\title{
Fractional-Order Generalized Predictive Control: Application for Low-Speed Control of Gasoline-Propelled Cars
}

\author{
M. Romero, ${ }^{1}$ A. P. de Madrid, ${ }^{1}$ C. Mañoso, ${ }^{1}$ V. Milanés, ${ }^{2}$ and B. M. Vinagre ${ }^{3}$ \\ ${ }^{1}$ Escuela Técnica Superior de Ingeniería Informática, UNED, Juan del Rosal, 16, 28040 Madrid, Spain \\ ${ }^{2}$ California PATH, University of California at Berkeley, Richmond, CA 94804-4698, USA \\ ${ }^{3}$ Industrial Engineering School, University of Extremadura, Avenida de Elvas s/n, 06071 Badajoz, Spain \\ Correspondence should be addressed to M. Romero; mromero@scc.uned.es
}

Received 9 November 2012; Accepted 22 January 2013

Academic Editor: Clara Ionescu

Copyright (c) 2013 M. Romero et al. This is an open access article distributed under the Creative Commons Attribution License, which permits unrestricted use, distribution, and reproduction in any medium, provided the original work is properly cited.

\begin{abstract}
There is an increasing interest in using fractional calculus applied to control theory generalizing classical control strategies as the PID controller and developing new ones with the intention of taking advantage of characteristics supplied by this mathematical tool for the controller definition. In this work, the fractional generalization of the successful and spread control strategy known as model predictive control is applied to drive autonomously a gasoline-propelled vehicle at low speeds. The vehicle is a Citroën C3 Pluriel that was modified to act over the throttle and brake pedals. Its highly nonlinear dynamics are an excellent test bed for applying beneficial characteristics of fractional predictive formulation to compensate unmodeled dynamics and external disturbances.
\end{abstract}

\section{Introduction}

Fractional calculus can be defined as a generalization of derivatives and integrals to noninteger orders, allowing calculations such as deriving a function to real or complex order $[1,2]$. Although this branch of mathematical analysis began 300 years ago when Liebniz and L'Hôpital discussed the possibility that $n$ could be a fraction $1 / 2$ for $n$th derivative $d^{n} y / d x^{n}$, it was really developed at the beginning of the 19th century by Liouville, Riemann, Letnikov, and other mathematicians [3].

Fractional-order operators are commonly represented by $D^{\alpha}$ that stands for $\alpha$-th-order derivative. Negative values of $\alpha$ correspond to fractional-order integrals: $D^{-\alpha} \equiv I^{\alpha}$. These operators can be evaluated using two general fractional definitions, Riemann-Liouville (RL) and GrünwaldLetnikov (GL). Both definitions, continuous and discrete, are equivalent for a wide class of functions which appear in real physical and engineering applications [1]. In this work, discrete domain will be exclusively considered. Hence, in the following the GL definition (1) will be used to implement fractional operators:

$$
D^{\alpha} f(t)_{t=k h}=\lim _{h \rightarrow 0} h^{-\alpha} \sum_{j=0}^{\infty}(-1)^{j}\left(\begin{array}{c}
\alpha \\
j
\end{array}\right) f(k h-j h), \quad \alpha \in \mathbb{R}
$$

where $\alpha$ is the fractional order of the derivative or integral, $h$ is the differential increment-close to zero-, and $j$ varies from 0 to $\infty$ due to the infinite memory of fractional operators.

In order to describe the dynamical behaviour of systems, the Laplace transform is often used. Expression (2) gives the Laplace transform of the GL definition under zero initial conditions. Nevertheless, the discretization of (2) does not lead to a transfer function with a limited number of coefficients in $z$ [4]. Thus, the so-called short memory principle [1] is applied, which means taking into account the behaviour only in the recent past that corresponds to a $n$-term truncated series, 


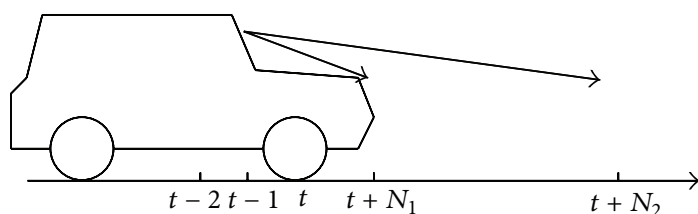

FIGURE 1: Model-based predictive control analogy.

paying a penalty in the form of some inaccuracy [5]:

$$
L\left\{D^{ \pm \alpha} f(t)\right\}=s^{ \pm \alpha} F(s), \quad \forall \alpha \in \mathbb{R} .
$$

Nowadays, this mathematical tool is more and more used in control theory to enhance the system performance. Typical fractional-order controllers include the CRONE control [6] and the $\mathrm{PI}^{\lambda} \mathrm{D}^{\mu}$ controller $[7,8]$. Advanced control system strategies have also been generalized: fractional optimal control [9-11], fractional fuzzy adaptive control [12], fractional nonlinear control [13], fractional iterative learning control [14], and fractional predictive control, the latter known as fractional-order generalized predictive control (FGPC), which was initially proposed in [15].

Model predictive control (MPC) is an advanced process control methodology in which a dynamical model of the plant is used to predict and optimize the future behaviour of the process over a time interval [16-18]. At each present time $t$, MPC generates a set of future control signals $u(t+k \mid t)$ based on the prediction of future process outputs $y(t+k \mid t)$ within the time window defined by $N_{1}$ (minimum costing horizon), $N_{2}$ (maximum costing horizon), and $N_{u}$ (control horizon). (With this notation, $x(t+k \mid t)$ stands for the value of $x$ at time $t+k$ predicted at time $t$.) However, only the first element of the control sequence $u(t \mid t)$ is applied to the system input. When the next measurement becomes available (present time equal to $t+1$ ), the previous procedure is repeated to find new predicted future process outputs $y(t+$ $1+k \mid t+1)$ and calculate the corresponding system input $u(t+1 \mid t+1)$ with prediction time windows moving forward; for this reason this kind of control is also known as receding horizon control (RHC). Figure 1 depicts the analogy between predictive control and a car driver who calculates the car manoeuvre following a receding horizon strategy [16].

MPC has become an industrial standard that has been widely adopted during the last 30 years. With over 2000 industrial installations, this control method is currently the most implemented for process plants [19]. It was originally developed to meet the specialized control needs of petroleum refineries [20, 21]. MPC technology can now be found in a wide variety of application areas such as chemicals [22, 23], solar power plants [24], agriculture [25], or clinical anaesthesia supply [26]. Recent developments related to MPC can be found in $[27,28]$.

Generalized predictive control (GPC) $[29,30]$ is one of the most representative MPC formulations. Its fractionalorder counterpart, FGPC, uses a real-order fractional cost function to combine the characteristics of fractional calculus and predictive control into a versatile control strategy [31-33].
On the other hand, driver-assistance systems have been a topic of active research during the last decades. They are intended to reduce traffic accidents and traffic congestions [34-37]. Open-loop cruise control (CC) systems are a wellknown class of driver-assistance systems, based on controlling the throttle pedal, that reduces driver workload and improve vehicle safety [38].

Nowadays, the tedious task of driving in traffic jams represents an unresolved issue in the automotive sector [39] because commercial vehicles exhibit highly nonlinear dynamics due to the behaviour of the vehicle engine at very low speed. Therefore, it constitutes one of the most important control challenges of the automotive sector [40]. Recently, approaches to resolve this problem have been studied both using experimental scaled-down vehicles [41] and using commercial vehicles $[42,43]$.

In this paper, an application of FGPC to the velocity control of a mass-produced car at very low speeds is described. The goal is to highlight the beneficial characteristics of FGPC to compensate unmodeled dynamics and external disturbances using the proposed tuning method. These characteristics were shown up in [32], where the lateral control of an autonomous vehicle is carried out by FGPC in the presence of sensor noise and the effect of the communication network.

The remainder of this paper is organized as follows: Section 2 summarizes the fundamentals of fractional predictive control methodology. Section 3 includes the description of the experimental vehicle, presents the design and tuning of the fractional predictive control, and shows the results of the experimental trial, including a comparison with integerorder GPC controllers. Finally, Section 4 draws the main conclusions of this work.

\section{Controller Formulation}

The GPC control law is obtained by minimizing, possibly subject to a set of constraints, the cost function:

$J_{\mathrm{GPC}}(\Delta u, t)=\sum_{k=N_{1}}^{N_{2}} \gamma_{k}(r(t+k)-y(t+k))^{2}+\sum_{k=1}^{N_{u}} \lambda_{k} \Delta u(t+k-1)^{2}$,

where $r$ is the reference, $y$ is the output, $u$ is the control signal, $\gamma_{k}$ and $\lambda_{k}$ are nonnegative weighting elements, $\Delta$ is the increment operator, and it is assumed that $u(t)$ remains constant from time instant $t+N_{u}\left(1 \leq N_{u} \leq N_{2}\right)[29,30]$. For the sake of simplicity in the notation $(\cdot \mid t)$ is omitted, since all expressions are referred to the present time $t$.

Outputs are predicted making use of a CARIMA model to describe the system dynamics:

$$
A\left(z^{-1}\right) y(t)=B\left(z^{-1}\right) u(t)+\frac{T_{c}\left(z^{-1}\right)}{\Delta} \xi(t),
$$

where $B\left(z^{-1}\right)$ and $A\left(z^{-1}\right)$ are the numerator and denominator of the model transfer function, respectively, $\xi(t)$ represents uncorrelated zero-mean white noise, and $T_{c}\left(z^{-1}\right)$ is a (pre)filter to improve the system robustness rejecting disturbance and noise $[44,45]$. 


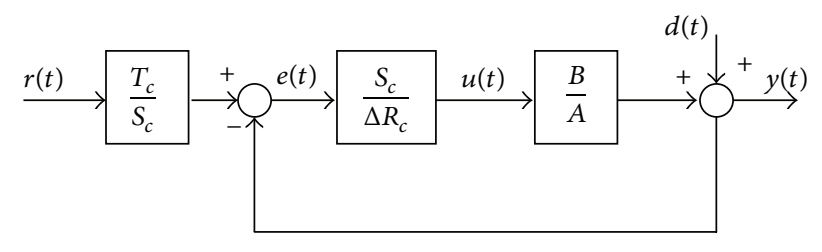

FIGURE 2: Closed-loop equivalent control schema.

Using model (4), the future system outputs $y(t+k)$ are predicted as $y=y_{C}+y_{F}$, where $y_{C}-$ forced response-is the part of the future output that depends on the future control actions $\Delta u$ (with $y_{C}=G \cdot \Delta u$, and $G$ the matrix of the step response coefficients of the model), and $y_{F}$-free responseis the part of the future output that does not depend on $\Delta u$ (i.e., the evolution of the process exclusively due to its present state) [29].

When no constraints are defined, the minimization of (3) leads to a linear time invariant (LTI) control law that can be precomputed in advance.

FGPC generalizes the GPC cost function (3) making use of the so-called fractional-order definite integration operator ${ }^{\alpha} I_{a}^{b}(\cdot)[15,46,47]$ (see the appendix):

$$
J_{\mathrm{FGPC}}(\Delta u, t)={ }^{\alpha} I_{\mathrm{N}_{1}}^{N_{2}}[e(t)]^{2}+{ }^{\beta} I_{1}^{N_{u}}[\Delta u(t-1)]^{2}, \quad \forall \alpha, \beta \in \mathbb{R},
$$

where $e \equiv r-y$ is the error. This cost function has been discretized with sampling period $\Delta t$ and evaluated using (A.2).

The FGPC cost function has an equivalent matrix form:

$$
J_{\mathrm{FGPC}}(\Delta u, t) \simeq e^{\prime} \Gamma(\alpha, \Delta t) e+\Delta u^{\prime} \Lambda(\beta, \Delta t) \Delta u,
$$

where $\Gamma$ and $\Lambda$ are infinite-dimensional square real weighting matrices which depend, by construction, on $\alpha$ and $\beta$, respectively:

$$
\Gamma \equiv \Delta t^{\alpha} \operatorname{diag}\left(\begin{array}{llllll}
\cdots & w_{n} & w_{n-1} & \cdots & w_{1} & w_{0}
\end{array}\right)
$$

with $w_{j}=\omega_{j}-\omega_{j-n}, n=N_{2}-N_{1}, \omega_{l}=(-1)^{l}\left(\begin{array}{c}-\alpha \\ l\end{array}\right)$, and $\omega_{l}=0$, for all $l<0$;

$$
\Lambda \equiv \Delta t^{\beta} \operatorname{diag}\left(\cdots \quad w_{N_{u}-1} \quad w_{N_{u}-2} \cdots w_{1} \quad w_{0}\right)
$$

with $w_{j}=\omega_{j}-\omega_{j-n}, n=N_{u}-1, \omega_{l}=(-1)^{l}\left(\begin{array}{c}-\beta \\ l\end{array}\right)$, and $\omega_{l}=0$, for all $l<0$.

In absence of constraints, the minimization of this cost function leads to a LTI control law similar to the one of GPC whose equivalent closed-loop schema is shown in Figure 2. See $[46,48]$ and the references therein for details.

$R_{c}$ and $S_{c}$ are the controller polynomials obtained from the model polynomials $A$ and $B$, and the controller parameters $N_{1}, N_{u}, N_{2}, \alpha$ and $\beta$, and $d$ stand for disturbance. From schema, it is easy to obtain

$$
R_{c} \Delta u(t)=T_{c} r(t)-S_{c} y(t) .
$$

The value of polynomials $R_{c}$ and $S_{c}$ is obtained using the expressions (10). $\Phi$ and $F$ are two polynomials obtained from the resolution of two Diophantine equations. See [16-18] for more details:

$$
\begin{gathered}
R_{c}\left(z^{-1}\right)=\frac{T_{c}\left(z^{-1}\right)+\sum_{i=N_{1}}^{N_{2}} k_{i} \Phi_{i}}{\sum_{i=N_{1}}^{N_{2}} k_{i} z^{-N_{2}+i}}, \\
S_{c}\left(z^{-1}\right)=\frac{\sum_{i=N_{1}}^{N_{2}} k_{i} F_{i}}{\sum_{i=N_{1}}^{N_{2}} k_{i} z^{-N_{2}+i}} .
\end{gathered}
$$

In GPC the weighting sequences $\gamma_{k}$ and $\lambda_{k}$ are controller parameters defined by the user. However, in FGPC these sequences are obtained from the optimization process itself and depend on the fractional integration orders $\alpha$ (7) and $\beta$ (8) as well as the controller horizons.

Tuning GPC and FGPC means setting the horizon parameters $\left(N_{1}, N_{u}, N_{2}\right)$ together with the weighting sequences $\gamma_{k}$ and $\lambda_{k}$ for GPC, and $\alpha$ and $\beta$ for FGPC, respectively. This task is critical because closed-loop stability depends on this choice. In GPC some thumb rules are usually accepted [29]. In FGPC, these thumb rules are also adequate for choosing the horizons $[15,46]$.

A FGPC-tuning method was proposed in [49]. Based on optimization, the objective is the system to fulfil phase margin, sensitivity functions, and some other robustness specifications. (This tuning method has already been used to tune fractional-order $\mathrm{PI}^{\lambda} \mathrm{D}^{\mu}$ controllers successfully [5052].) In order to keep the dimension of the optimization problem low, it is assumed that the horizon parameters $\left(N_{1}, N_{u}, N_{2}\right)$ are given (for instance, following the thumbrules previously announced), and only the two unknown parameters, the fractional orders $\alpha$ and $\beta$, are used in the optimization process. Thus, the function FMINCON of the MATLAB optimization toolbox [53] can be used to solve the corresponding optimization problem.

\section{Experimental Application}

In this section, we present a practical application of FGPC. We describe its design, tuning, and practical performance on the longitudinal speed control of a commercial vehicle.

3.1. Experimental Vehicle. The vehicle used for the experimental phase is a convertible Citroën C3 Pluriel (Figure 3) which is equipped with automatic driving capabilities by means of hardware modifications to permit autonomous actions on the accelerator and brake pedals. These modifications let the controller's outputs steer the vehicle's actuators.

The car's throttle is handled by an analog signal that represents the pressure on the pedal, generated by an analog card. The action over the throttle pedal is transformed into two analogue values-one of them twice the other-between 0 and $5 \mathrm{~V}$. A switch has been installed on the dashboard to commute between automatic throttle control and original throttle circuit.

The brake's automation has been done taking into account that its action is critical. In case of a failure of any of the autonomous systems, the vehicle can be stopped by human driver intervention. So an electrohydraulic braking system 


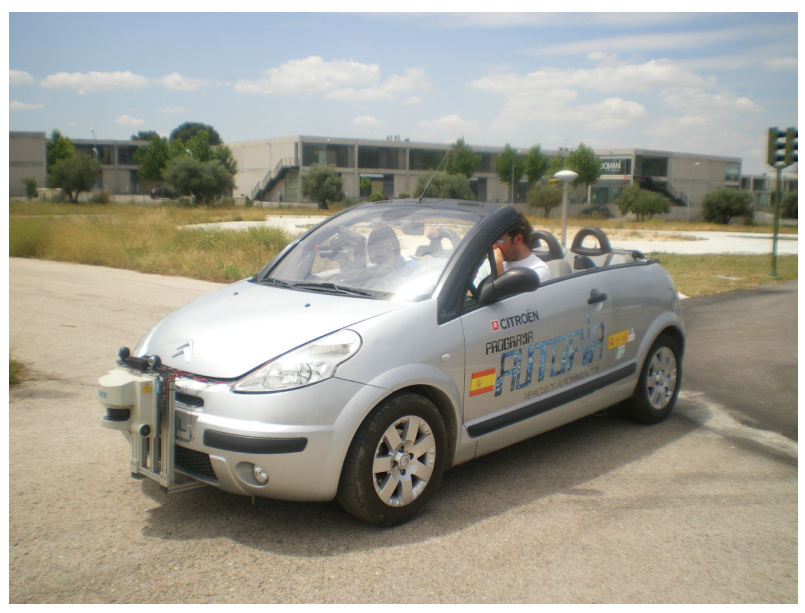

FIgURE 3: Commercial Citroën C3 prototype vehicle.

is mounted in parallel with the original one, permitting to coexist the two braking system independently. More details about throttle and brake automation can be found in $[54,55]$.

Concerning the on-board sensor systems, a real-time kinematic-differential global positioning system (RTKDGPS) that gives vehicle position with a 1 centimeter precision and an inertial unit (IMU) to improve the positioning when GPS signal fails are used to obtain the vehicle's true position. The car's actual speed and acceleration are obtained from a differential hall effect sensor and a piezoelectric sensor, respectively. These values are acquired via controller area network bus (CAN) and provide the necessary information to the control algorithm, which is running in real-time in the on-board control unit (OCU), generating the control actions to govern the actuators.

For the purpose of this work, the gearbox is always in first gear forcing the car to move at low speed. The sampling interval was fixed by the parameters of GPS at $200 \mathrm{~ms}$. Therefore, the frequency of actions on the pedals is set to $5 \mathrm{~Hz}$. Using these settings, the OCU can approximately perform an action every metre at a maximum speed of $20 \mathrm{~km} / \mathrm{h}$.

3.2. Identification of the Longitudinal Dynamics. Due to the gasoline-propelled vehicle dynamics at very low speeds are highly nonlinear, and finding an exact dynamical model for the vehicle is not an easy task. Nevertheless, as we have seen previously, fractional predictive controller needs a CARIMA model of the plant to make the predictions. Therefore, an identification process has to be carried out despite inevitable uncertainties and circuit perturbations.

Since the vehicle always remains in first gear, restricting its speed at less than $20 \mathrm{~km} / \mathrm{h}$ and acting a high engine brake force, the identification process is only fulfilled for the throttle pedal. Taking the brake pedal effect into account leads us to a hybrid control strategy that is not the purpose of this paper.

The experimental vehicle response is shown in Figure 4 (solid line), where the vehicle has been subjected to several speed changes by means of successive throttle pedal actuations. (In Figure 4, the action of the brake pedal is also depicted but is not taken into consideration in the identification process; it has been used for the purpose of returning to the initial speed, $0 \mathrm{~km} / \mathrm{h}$.)

The model of the vehicle is obtained by means of an identification process using the MATLAB Identification Toolbox [56], considering a normalized input-in the interval $(0,1)$ for the throttle pedal and the sampling time of GPS fixed at $200 \mathrm{~ms}$ :

$$
G\left(z^{-1}\right)=\frac{5.1850 z^{-4}}{1-0.7344 z^{-1}-0.2075 z^{-2}} .
$$

The time-domain model validation is depicted in Figure 4. It is observable that model (11) captures the vehicle dynamics reasonably good (dash line) in comparison with the experimental data (solid line), despite environment and circuit perturbations.

3.3. Controller Design. This section describes the controller design for the longitudinal speed control of the vehicle described previously. Transfer function (11) constitutes the starting point in the controller tuning, where beneficial characteristics of fractional predictive formulation will be used to compensate unmodeled dynamics and external disturbances.

Other practical requirements have to be taken into account during the design process. (1) The car response has to be smooth to guarantee that its acceleration is less than $\pm 2 \mathrm{~m} / \mathrm{s}^{2}$, the maximum acceptable acceleration for standing passengers [57]. (2) Control action $u$ is normalized and has to be in the interval $[0,1]$, where negative values are not allowed as they mean brake actions.

Firstly, the horizons are chosen to capture the loop dominant dynamics. We have taken a time window of 2 seconds ahead defined by $N_{1}=1$ and $N_{2}=10$, which is appropriated in a heavy traffic scene (low speed). A wider time window supposes an increment of $N_{2}$ that would lead to a system with an excessively slow response. On the other hand, we have also considered the control horizon $N_{u}=$ 2 , which represents an agreement between system response speed and comfort of the vehicle's occupants. It is well-known that larger values of $N_{u}$ produce tighter control actions [16] that could even make the system unstable.

Moreover, we have used a prefilter $T_{c}\left(z^{-1}\right)$ to improve the system robustness against the model-process mismatch and the disturbance rejection. In [44] a guideline is given:

$$
T_{c}\left(z^{-1}\right)=\left(1-\rho z^{-1}\right)^{N_{1}}
$$

where $\rho$ is recommended to be close to the dominant pole of (11).

Thus, the chosen prefilter has the following expression:

$$
T_{c}\left(z^{-1}\right)=1-0.9 z^{-1}
$$

Once the controller horizons and the prefilter are chosen, the objective of the optimization process is finding the pair $(\alpha, \beta)$ that fulfils some specified robustness criteria. In our case, we shall impose the following.

(i) Maximize the phase margin (no specification is set on the gain margin). 


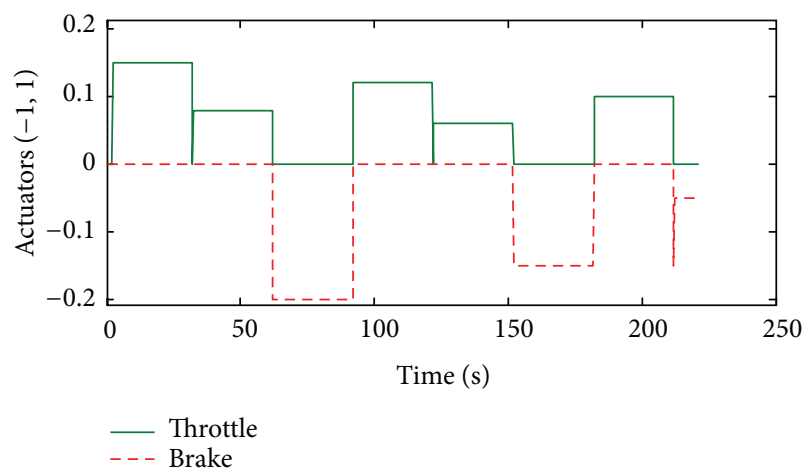

(a)

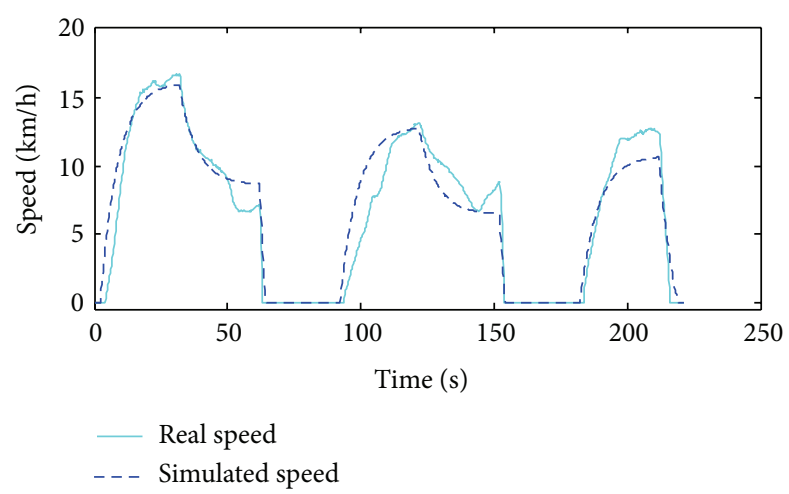

(b)

FIGURE 4: Experimental vehicle response and time-domain model validation.

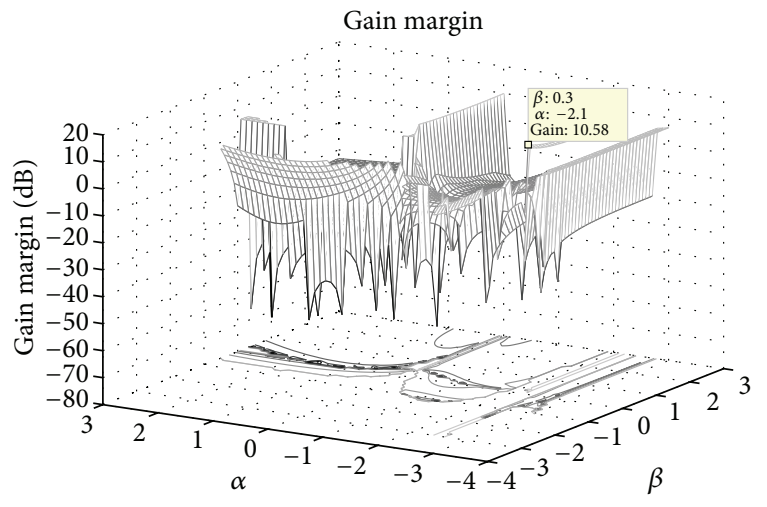

FIGURE 5: FGPC gain margin versus $\alpha$ and $\beta$.

(ii) Sensitivity function $|S(j \omega)| \leq-30 \mathrm{~dB}$ for $\omega \leq 0.01 \mathrm{rad} /$ s.

(iii) Complementary sensitivity function $|T(j \omega)| \leq 0 \mathrm{~dB}$ for $\omega \geq 0.1 \mathrm{rad} / \mathrm{s}$.

(Phase margin maximization guarantees smooth system output and robustness; sensitivity functions constraints give good noise and disturbance rejection.)

In order to initialize the optimization algorithm an initial seed $\left(\alpha_{0}, \beta_{0}\right)$ is needed. Figures 5 and 6 depict the closed loop magnitude and phase margins, respectively, in the interval $\alpha, \beta \in[-3,3]$. We select $\alpha_{0}=-2.1$ and $\beta_{0}=0.3$ for their corresponding good gain and phase margins.

The optimization process has been carried out in an interval of 20-30 seconds using a PC computer with Intel Core 2 Duo T9300 $2.5 \mathrm{GHz}$ running MATLAB 2007a. The solution to the optimization problem is $\alpha^{*}=-2.2456$ and $\beta^{*}=2.9271$, for which the weighting sequences $\Gamma$ and $\Lambda$ are given in (14), with a phase margin of $76.76^{\circ}$ (and a gain margin of $15.51 \mathrm{~dB}$ ). The controller sensitivity functions meet the design specifications, as it is depicted in Figure 7:

$$
\begin{aligned}
& \Gamma=\operatorname{diag}\left(\begin{array}{lllll}
-36.9671 & -0.0406 & -0.0683 & -0.1273 & -0.1273
\end{array}\right. \\
& \begin{array}{lllll}
-0.7881 & -0.7881 & 51.4711 & 82.8442 & 36.9411)
\end{array} \\
& \Lambda=\operatorname{diag}\left(\begin{array}{lll}
0.0173 & 0.0090
\end{array}\right) .
\end{aligned}
$$

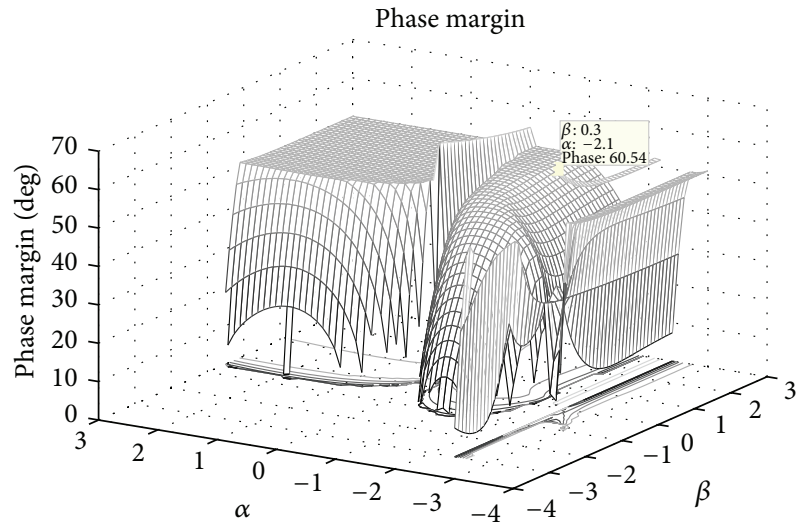

FIGURE 6: FGPC phase margin versus $\alpha$ and $\beta$.

3.4. Experimental Results. The experimental trial was accomplished at the Centre for Automation and Robotics (CAR; joint research centre by the Spanish Consejo Superior de Investigaciones Científicas and the Universidad Politécnica de Madrid) private driving circuit using the Citroën C3 Pluriel described previously. The circuit has been designed with scientific purposes and represents an inner-city area with straight-road segments, bends, and so on. Figure 8 shows an aerial sight.

To validate the proposed controller, various target speed changes were set each 25 seconds, trying to keep the speed error close to zero. Moreover, the automatic gearbox was always in first gear, avoiding any effect of gear changes and forcing the car to move at low speed. Figure 9 depicts the responses of the vehicle, both actual-real time-(dot line) and simulated (dash-dot line). The FGPC controller accomplished all practical requirements which were set previously. The vehicle response is stable, smooth, and reasonably good in comparison with its simulation. It is important to remark that the positive reference changes are faster than the negative one. This is mainly due to the fact that the braking manoeuvre has to be achieved by the engine brake force, and it is affected by the slope of the circuit.

With respect to the comfort of the vehicle's occupants, it is observable that vehicle acceleration always remains (in 


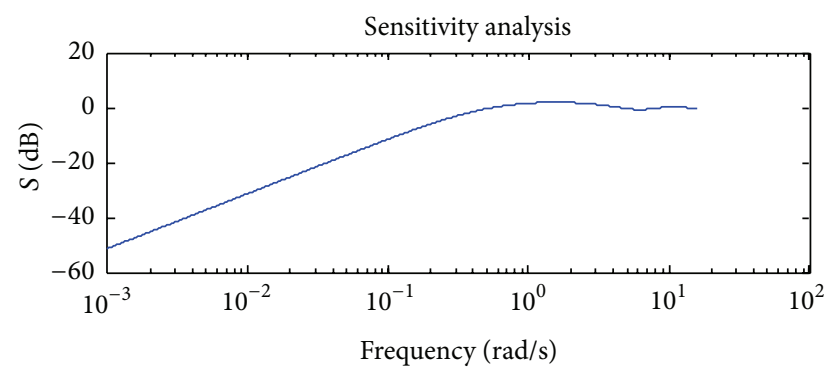

(a)

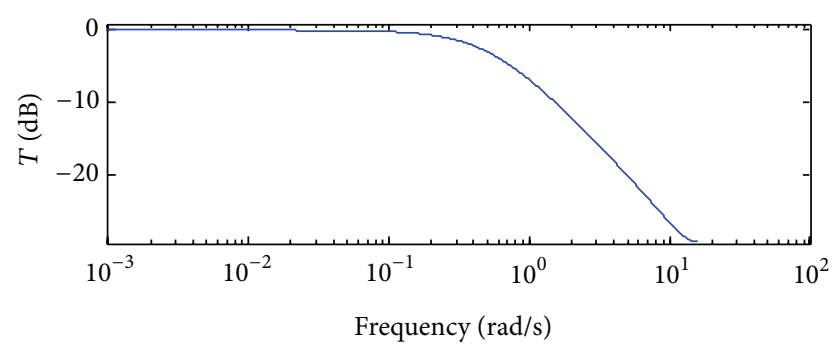

(b)

FIgURE 7: Sensitivity functions.

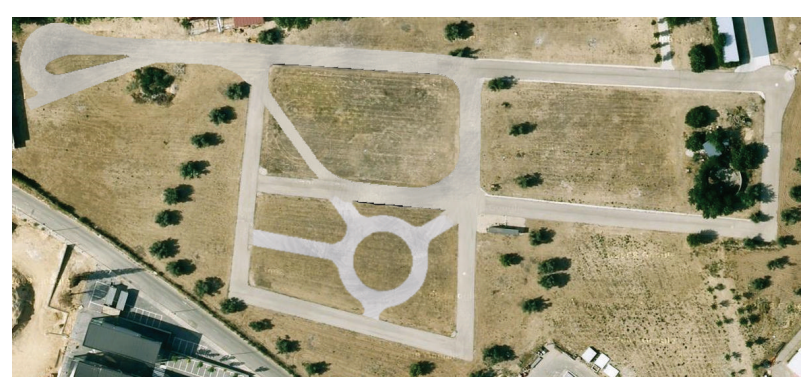

FIgURE 8: Private driving circuit at CAR.

absolute value) below the maximum acceptable acceleration requirement, $2 \mathrm{~m} / \mathrm{s}^{2}$. It is due to the soft action over the throttle vehicle actuator, satisfying the comfort driving requisites.

For comparison purposes, we have also tested the performance of several GPCs which were tuned using the same horizons $\left(N_{1}=1, N_{u}=2\right.$, and $\left.N_{2}=10\right)$ and prefilter $T_{c}(13)$ as FGPC.

In practice, in GPC it is commonly assumed that the weighting sequences are constant, that is, $\gamma_{k}=\gamma$ and $\lambda_{k}=$ $\lambda$. Under this assumption, it has not been possible to find a GPC controller that fulfils the robustness criteria using and equivalent optimization method. (The set of dynamics that can be found with constant weights is much smaller than in the case of FGPC. Furthermore, trying to optimize a GPC controller in the general case $\left(\gamma_{k}, \lambda_{k}\right)$ would lead to an optimization problem with an extremely high dimension. On the other hand, in the case of FGPC one has to optimize only two parameters, $\alpha$ and $\beta$, and this automatically leads to nonconstant weighting sequences; recall that GPC and FGPC controllers share a common LTI expression, as was pointed out in Section 2 [49].)

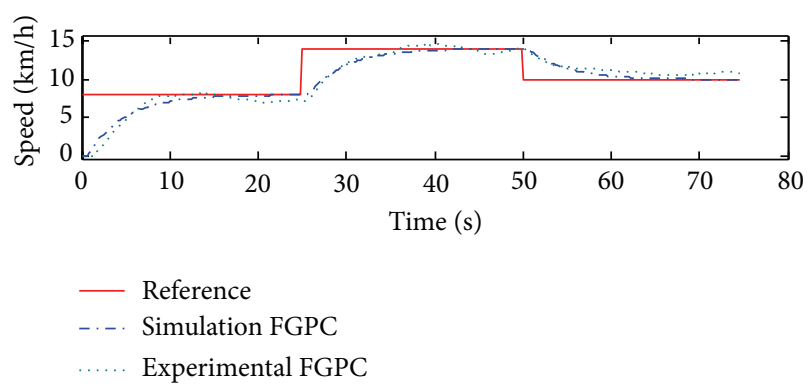

(a)

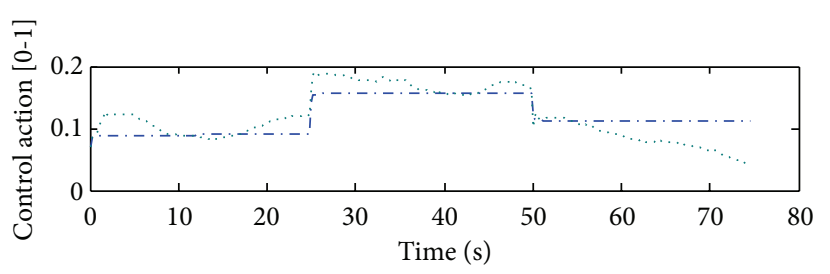

(b)

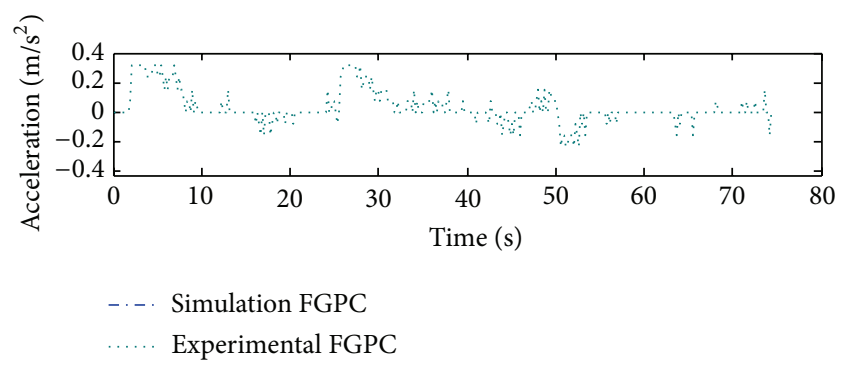

(c)

FIGURE 9: FGPC controller performance.

For this reason, we have tuned several GPC controllers with different constant weighting sequences $\gamma$ and $\lambda$. Specifically, $\lambda \in\left\{10^{-6}, 10^{-1}, 10^{1}, 10^{5}\right\}$ and $\gamma=1$ (as the variation of $\gamma$ does not affect the system dynamics considerably).

Using these settings, we have obtained two GPC controllers that in practice turned out to be unstable although they were stable in simulation. These controllers correspond to $\lambda=10^{-6}$ and $\lambda=10^{-1}$ (labelled Experimental GPC 1 and Experimental GPC 2 in Figure 10, resp.). Thus, they were not able to compensate unmodeled dynamics and circuit perturbations.

On the other hand, GPC controllers for $\lambda=10^{1}$ and $\lambda=$ $10^{5}$ (labelled Experimental GPC 3 and Experimental GPC 4 in Figure 11, resp.) were stable in practice. It is well-known that higher values of $\lambda$ give rise to smooth control actions, increasing the closed loop system robustness [16]. However, an excessively high value of $\lambda$ could make the system response too slow. It would mean, in practice, that our car could not stop in time, and it would probably crash into the front car.

To quantify these results, we shall compare the principal control quality indicators for the stable realizations (GPC 3, GPC 4, and FGPC) speed error (reference speedexperimental speed), softness of the control action, and acceleration. The last ones require to calculate the fast fourier transform (FFT) to estimate them. 


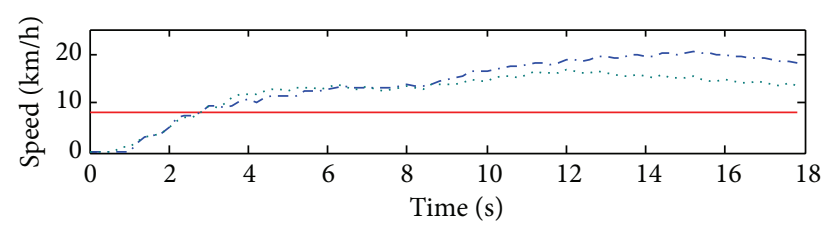

- Reference

-.-. Experimental GPC 1 Experimental GPC 2

(a)

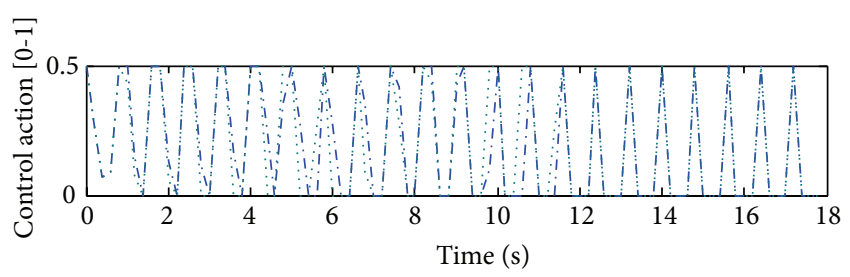

(b)

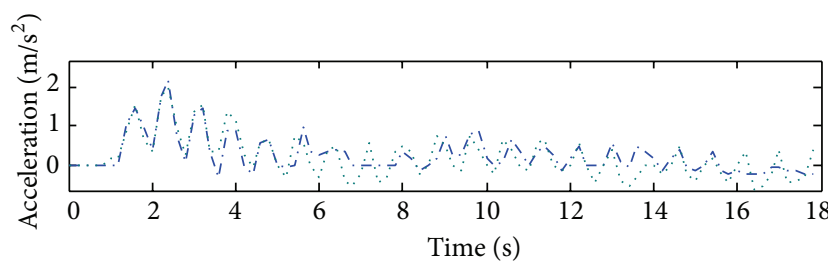

-.. . Experimental GPC 1

Experimental GPC 2

(c)

FIgURE 10: Unstable GPC controllers. Action over the throttle has been limited to [0-0.5] for passengers safety during the experimental trial.

It is well known that FFT (15) is an efficient algorithm to compute the discrete fourier transform (DFT), $\mathfrak{~}$

$$
U_{k}=\mathfrak{F}\left(u_{k}\right)=\sum_{i=0}^{N-1} u_{k} e^{\left(2 \pi N / k_{i}\right)}, \quad k=0, \ldots, N-1,
$$

where $u_{k}$ is the control action or acceleration value at time $t_{k}$ and $N$ the length of these signals. FFT yields the signal sharpness by means of a frequency spectrum analysis of the sampled signal.

In order to get a good indicator of the overall control action and acceleration signals with robustness to outliers, we have used the median $\widetilde{u}$ of sequence $U_{k}$.

$$
P\left(U_{k} \leq \tilde{u}\right) \geq \frac{1}{2} \wedge P\left(U_{k} \geq \tilde{u}\right) \geq \frac{1}{2} .
$$

The following widely used statistics parameters have been used to evaluate the speed error:

(i) mean:

$$
\bar{e}=\frac{1}{N} \sum_{i=0}^{N-1} e_{i}
$$

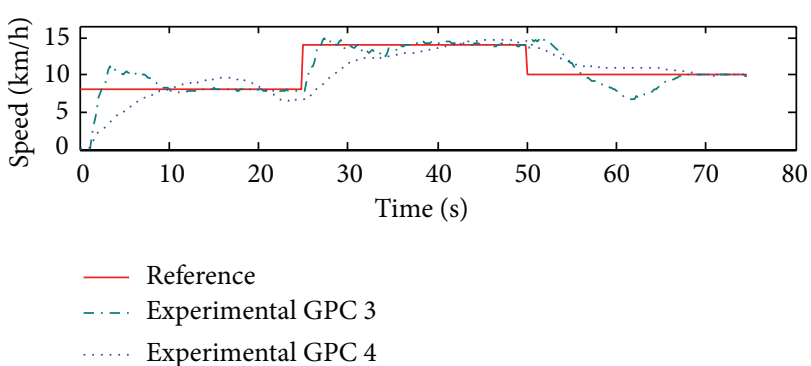

(a)

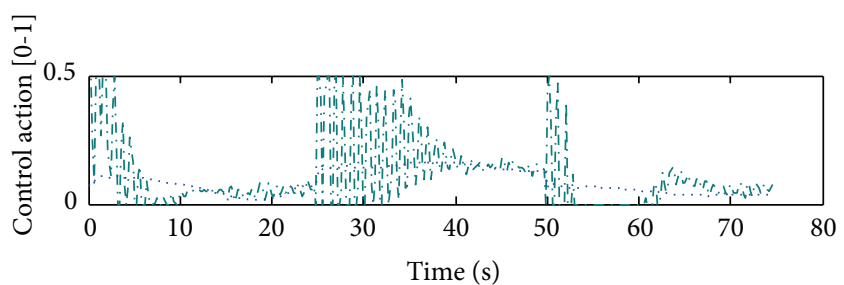

(b)

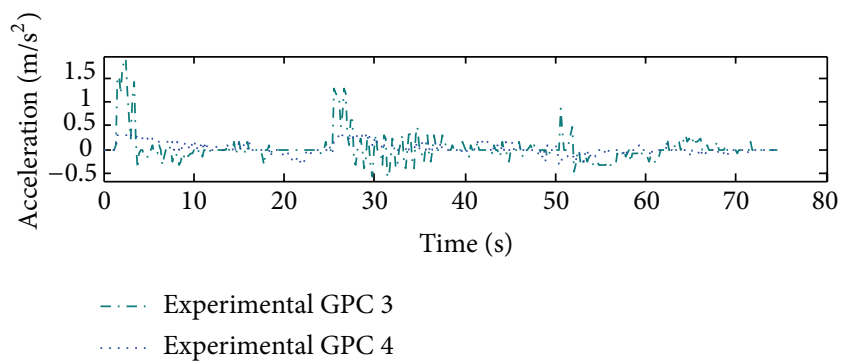

(c)

FIGURE 11: Stable GPC controllers.

(ii) standard deviation:

$$
\sigma=\sqrt{\frac{1}{N} \sum_{i=0}^{N-1}\left(e_{i}-\bar{e}\right)^{2}}
$$

(iii) root mean square error:

$$
\mathrm{RMSE}=\sqrt{\frac{1}{N} \sum_{i=0}^{N-1} e_{i}^{2}}
$$

where $e_{k}$ is the speed error at time $t_{k}$. Moreover, we have also used the median $\tilde{e}$.

All of these control quality indicators are reflected in Table 1.

One observes (see Figures 9 and 11) that the speed changes of GPC 4 and FGPC are slower than the response of GPC 3 , so they need more time to reach the steady state after speed changes. This is reflected in Table 1 where, in terms of speed error, all statistics parameters of GPC 3 are better than the GPC 4 and FGPC ones. However, it presents very poor values in the control action and acceleration indicators due to the very large fluctuations of these signals, as we can see graphically in Figure 11. This undesirable behaviour 
TABLE 1: Comparison of stable controllers.

\begin{tabular}{|c|c|c|c|c|c|c|}
\hline \multirow{2}{*}{ Contr. } & \multicolumn{4}{|c|}{ Speed error } & \multirow{2}{*}{$\begin{array}{l}\text { Control action } \\
\text { FFT median }\end{array}$} & \multirow{2}{*}{$\begin{array}{l}\text { Acceleration } \\
\text { FFT median }\end{array}$} \\
\hline & Mean & St. dev. & Median & RMSE & & \\
\hline GPC 3 & 0.0474 & 2.1340 & 2.5539 & 2.1317 & 0.4397 & 1.9320 \\
\hline GPC 4 & 0.3955 & 2.5186 & 6.3434 & 2.5461 & 0.0149 & 1.0562 \\
\hline FGPC & 0.4604 & 2.4119 & 5.8174 & 2.4523 & 0.0044 & 0.1652 \\
\hline
\end{tabular}

compromises seriously the comfort of standing passengers, bordering on the maximum acceptable acceleration, $2 \mathrm{~m} / \mathrm{s}^{2}$. Furthermore, it could injurey the throttle actuator due to its continuous and aggressive fluctuations in the control action.

The FGPC controller shows the best behaviour in the steady state without overshoot and presenting the best values in terms of the softness of the control action and acceleration, due to the precise parameters tuning carried out by the optimization method. FGPC takes advantage of its diversity of responses (varying the fractional orders $\alpha$ and $\beta$ ) to meet the design specifications and to improve the system robustness against the model-process mismatch.

\section{Conclusions}

The longitudinal control of a gasoline-propelled vehicle at low speeds (common situation in traffic jams) constitutes one of the most important topics in the automotive sector due to the highly nonlinear dynamics that the vehicle presents in this situation.

In this paper, the fractional predictive control strategy, FGPC, has been used to solve this problem. Taking advantage of its beneficial characteristics and its tuning method to compensate un-modeled dynamics, a FGPC controller has been designed which has achieved closed loop stability following the changes in the velocity reference. Moreover, practical requirements to guarantee standing passengers comfort have been also achieved by means of the appropriate parameters choice carried out by the optimization-based tuning, in spite of inevitable uncertainties and circuit perturbations.

Finally, the comparison between the fractional predictive control strategy, FGPC, and its integer-order counterpart, GPC, has shown that the task of finding the correct setting for the weighting sequences $\gamma_{k}$ and $\lambda_{k}$ is crucial. In FGPC, the fractional orders $\alpha$ and $\beta$ allow us to find them keeping the dimension of the optimization problem low, since only two parameters have been optimized.

\section{Appendix}

\section{Fractional-Order Definite Integral Operator}

The fractional-order definite integral of function $f(x)$ within interval $[a, b]$ has the following expression [47]:

$$
{ }^{\alpha} I_{a}^{b} f(x) \equiv \int_{a}^{b}\left[D^{1-\alpha} f(x)\right] d x, \quad \alpha, a, b \in \mathbb{R}
$$

Using the GL definition (1) assuming that $D^{1-\alpha}[f(x)] \neq 0$, the fractional-order definite integrator operator ${ }^{\alpha} I_{a}^{b}(\cdot)$ has the following discretized expression with a sampling period $\Delta t$ :

$$
{ }^{\alpha} I_{a}^{b} f(x)=\Delta x^{\alpha} \bar{W}^{\prime} \bar{f}
$$

where

$$
\begin{aligned}
& \bar{W}=\left(\begin{array}{lllllllll}
\cdots & w_{b} & w_{b-1} & \cdots & w_{n+1} & w_{n} & \cdots & w_{1} & w_{0}
\end{array}\right)^{\prime} \\
& \bar{f}=\left(\begin{array}{lllll}
\cdots & f(0) & f(\Delta x) & \cdots & f(a-\Delta x)
\end{array} f(a)\right. \\
& \cdots f(b-\Delta x) f(b))^{\prime}
\end{aligned}
$$

with $w_{j}=\omega_{j}-\omega_{j-n}, n=b-a, \omega_{l}=(-1)^{l}\left(\begin{array}{c}-\alpha \\ l\end{array}\right)$, and $\omega_{l}=0$, for all $l<0$.

\section{Conflicts of Interest}

The authors report no actual or potential conflict of interests in relation to this manuscript.

\section{Acknowledgments}

The authors wish to acknowledge the economical support of the Spanish Distance Education University (UNED), under Project reference PROY29 (Proyectos Investigación 2012), and the AUTOPIA Program of the Center for Automation and Robotics UPM-CSIC.

\section{References}

[1] I. Podlubny, Fractional Differential Equations, vol. 198 of Mathematics in Science and Engineering, Academic Press, San Diego, Calif, USA, 1999.

[2] K. B. Oldham and J. Spanier, The Fractional Calculus, vol. 111 of Mathematics in Science and Engineering, Academic Press, New York, NY, USA, 1974.

[3] K. S. Miller and B. Ross, An Introduction to the Fractional Calculus and Fractional Differential Equations, John Wiley \& Sons, New York, NY, USA, 1993.

[4] B. M. Vinagre, C. A. Monje, and A. J. Calderón, "Fractional order systems and fractional order control actions," in Proceedings of the 41st Conference on Decision and Control. Tutorial Workshop 2: Fractional Calculus Applications in Automatic Control and Robotics, Las Vegas, Nev, USA, 2002.

[5] I. Podlubny, "Numerical solution of ordinary fractional differential equations by the fractional difference method," in Advances in Difference Equations (Veszprém, 1995), pp. 507-515, Gordon and Breach, Amsterdam, The Netherlands, 1997. 
[6] A. Oustaloup, B. Mathieu, and P. Lanusse, "The CRONE control of resonant plants: application to a flexible transmission," European Journal of Control, vol. 1, pp. 113-121, 1995.

[7] I. Podlubny, "Fractional-order systems and $P I^{\lambda} D^{\mu}$-controllers," IEEE Transactions on Automatic Control, vol. 44, no. 1, pp. 208214, 1999.

[8] I. Petráš, “The fractional-order controllers," Journal of Electrical Engineering, vol. 50, pp. 284-288, 1999.

[9] O. P. Agrawal, "A general formulation and solution scheme for fractional optimal control problems," Nonlinear Dynamics, vol. 38, no. 1-4, pp. 323-337, 2004.

[10] O. P. Agrawal and D. Baleanu, "A Hamiltonian formulation and a direct numerical scheme for fractional optimal control problems," Journal of Vibration and Control, vol. 13, no. 9-10, pp. 1269-1281, 2007.

[11] C. Tricaud and Y. Q. Chen, "Solving fractional order optimal control problms in RIOTS_95-a general purpose optimal control problems solver," in Proceedings of the 3rd IFAC Workshop on Fractional Differentiation and Its Applications, Ankara, Turkey, 2008.

[12] M. O. Efe, "Fractional fuzzy adaptive sliding-mode control of a 2-DOF direct-drive robot arm," IEEE Transactions on Systems, Man, and Cybernetics, Part B, vol. 38, no. 6, pp. 1561-1570, 2008.

[13] I. S. Jesus, J. T. Machado, and R. S. Barbosa, "Fractional order nonlinear control of heat system," in Proceedings of the 3rd IFAC Workshop on Fractional Differentiation and Its Applications, Ankara, Turkey, 2008.

[14] Y. Li, Y. Chen, and H.-S. Ahn, "Fractional-order iterative learning control for fractional-order linear systems," Asian Journal of Control, vol. 13, no. 1, pp. 54-63, 2011.

[15] M. Romero, A. P. de Madrid, C. Mañoso, and R. Hernandez, "Generalized predictive control of arbitrary real order," in New Trends in Nanotechnology and Fractional Calculus Applications, D. Baleanu, Z. B. Guvenc, and J. A. T. Machado, Eds., pp. 411418, Springer, Dordrecht, The Netherlands, 2009.

[16] E. F. Camacho and C. Bordons, Model Predictive Control, Springer, New York, NY, USA, 2nd edition, 2004.

[17] J. A. Rossiter, Model Based Predictive Control. A Practical Approach, CRC Press, New York, NY, USA, 2003.

[18] J. M. Maciejowski, Predictive Control with Constraints, Prentice Hall, New York, NY, USA, 2002.

[19] J. Qin and T. Badgwell, "An overview of industrial model predictive control technology," in Proceedings of the International Conference on Chemical Process, J. C. Kantor, C. E. Garcia, and B. Carnahan, Eds., vol. 93 of AIChE Symposium Series, pp. 232256, 1997.

[20] C. R. Cutler and B. L. Ramaker, "Dynamic matrix control-a computer control algorithm," in Proceedings of Joint Automatic Control Conference, San Francisco, Calif, USA, 1980.

[21] W. L. Luyben, Ed., Practical Distillation Control, Van Nostrand Reinhold, New York, NY, USA, 1992.

[22] T. Alvarez, M. Sanzo, and C. de Prada, "Identification and constrained multivariable predictive control of chemical reactors," in Proceedings of the IEEE Conference on Control Applications, pp. 663-664, Albany, NY, USA, September 1995.

[23] J. M. Martín Sánchez and J. Rodellar, Adaptive Predictive Control. From the Concepts to Plant Optimization, Prentice Hall, Upper Saddle River, NJ, USA, 1996.

[24] E. F. Camacho and M. Berenguel, "Application of generalized predictive control to a solar power plant," in Proceedings of the IEEE Conference on Control Applications, pp. 1657-1662, Glasgow, UK, August 1994.

[25] F. Han, C. Zuo, W. Wu, J. Li, and Z. Liu, "Model predictive control of the grain drying process," Mathematical Problems in Engineering, vol. 2012, Article ID 584376, 12 pages, 2012.

[26] D. A. Linkens and M. Mahfouf, "Generalized predictive control (GPC) in clinical anaesthesia," in Advances in Model-Based Predictive Control, D. W. Clarke, Ed., pp. 429-445, Oxford University Press, Oxford, UK, 1994.

[27] H. Yang and S. Li, "A data-driven bilinear predictive controller design based on subspace method," Asian Journal of Control, vol. 13, no. 2, pp. 345-349, 2011.

[28] X.-H. Chang and G.-H. Yang, "Fuzzy robust constrained model predictive control for nonlinear systems," Asian Journal of Control, vol. 13, no. 6, pp. 947-955, 2011.

[29] D. W. Clarke, C. Mohtadi, and P. S. Tuffs, "Generalized predictive control. Part I. The basic algorithm," Automatica, vol. 23, no. 2, pp. 137-148, 1987.

[30] D. W. Clarke, C. Mohtadi, and P. S. Tuffs, "Generalized predictive control. Part II. Extensions and interpretations," Automatica, vol. 23, no. 2, pp. 149-160, 1987.

[31] M. Romero, I. Tejado, B. M. Vinagre, and A. P. de Madrid, "Position and velocity control of a servo by using GPC of arbitrary real order," in New Trends in Nanotechnology and Fractional Calculus Applications, D. Baleanu, Z. B. Guvenc, and J. A. T. Machado, Eds., pp. 369-376, Springer, Dordrecht, The Netherlands, 2009.

[32] M. Romero, I. Tejado, J. I. Suárez, B. M. Vinagre, and A. P. De Madrid, "GPC strategies for the lateral control of a networked AGV," in Proceedings of the 5th International Conference on Mechatronics (ICM '09), Málaga, Spain, April 2009.

[33] I. Tejado, M. Romero, B. M. Vinagre, A. P. de Madrid, and Y. Q. Chen, "Experiences on an internet link characterization and networked control of a smart wheel," International Journal of Bifurcation and Chaos, vol. 22, no. 4, 2012.

[34] R. Bishop, "A survey of intelligent vehicle applications worldwide," in Proceedings of the IEEE Intelligent Vehicles Symposium, pp. 25-30, Dearborn, Mich, USA, 2000.

[35] G. Marsden, M. McDonald, and M. Brackstone, "Towards an understanding of adaptive cruise control," Transportation Research Part C, vol. 9, no. 1, pp. 33-51, 2001.

[36] A. Vahidi and A. Eskandarian, "Research advances in intelligent collision avoidance and adaptive cruise control," IEEE Transactions on Intelligent Transportation Systems, vol. 4, no. 3, pp. 132153, 2003.

[37] B. Van Arem, C. J. G. Van Driel, and R. Visser, “The impact of cooperative adaptive cruise control on traffic-flow characteristics," IEEE Transactions on Intelligent Transportation Systems, vol. 7, no. 4, pp. 429-436, 2006.

[38] T. Aono and T. Kowatari, "Throttle-control algorithm for improving engine response based on air-intake model and throttle-response model," IEEE Transactions on Industrial Electronics, vol. 53, no. 3, pp. 915-921, 2006.

[39] N. B. Hounsell, B. P. Shrestha, J. Piao, and M. McDonald, "Review of urban traffic management and the impacts of new vehicle technologies," IET Intelligent Transport Systems, vol. 3, no. 4, pp. 419-428, 2009.

[40] S. Moon, I. Moon, and K. Yi, "Design, tuning, and evaluation of a full-range adaptive cruise control system with collision avoidance," Control Engineering Practice, vol. 17, no. 4, pp. 442455, 2009. 
[41] L. Cai, A. B. Rad, and W. L. Chan, "An intelligent longitudinal controller for application in semiautonomous vehicles," IEEE Transactions on Industrial Electronics, vol. 57, no. 4, pp. 14871497, 2010.

[42] V. Milanés, J. Villagrá, J. Godoy, and C. González, "Comparing fuzzy and intelligent PI controllers in stop-and-go manoeuvres," IEEE Transactions on Control Systems Technology, vol. 20, no. 3, pp. 770-778, 2011.

[43] I. Tejado, V. Milanés, J. Villagrá, J. Godoy, H. HosseinNia, and B. M. Vinagre, "Low speed control of an autonomous vehicle by using a fractional PI," in Proceedings of the 18th World Congress International Federation Automatic Control, pp. 15025-15030, Milano, Italy, 2011.

[44] T.-W. Yoon and D. W. Clarke, "Observer design in recedinghorizon predictive control," International Journal of Control, vol. 61, no. 1, pp. 171-191, 1995.

[45] C. Mañoso, A. P. de Madrid, M. Romero, and R. Hernández, "GPC with structured perturbations: the influence of prefiltering and terminal equality constraints," ISRN Applied Mathematics, vol. 2012, Article ID 623484, 12 pages, 2012.

[46] M. Romero, A. P. de Madrid, C. Mañoso, and B. M. Vinagre, "Fractional-order generalized predictive control: formulation and some properties," in proceedings of the 11th International Conference on Control, Automation, Robotics and Vision (ICARCV '10), pp. 1495-1500, Singapore, December 2010.

[47] M. Romero, A. P. de Madrid, and B. M. Vinagre, "Arbitrary realorder cost functions for signals and systems," Signal Processing, vol. 91, no. 3, pp. 372-378, 2011.

[48] M. Romero, A. P. de Madrid, C. Mañoso, and B. M. Vinagre, "A survey of fractional-order generalized predictive control," in Proceedings of the 51st Conference on Decision and Control, pp. 6867-6872, Maui, Hawaii, USA, 2012.

[49] M. Romero, I. Tejado, A. P. de Madrid, and B. M. Vinagre, "Tuning predictive controllers with optimization: application to GPC and FGPC," in Proceedings of the 18th World World Congress International Federation Automatic Control, pp. 1082410829, Milano, Italy, 2011.

[50] C. A. Monje, A. J. Calderón, B. M. Vinagre, Y. Chen, and V. Feliu, "On fractional $P I^{\lambda}$ controllers: some tuning rules for robustness to plant uncertainties," Nonlinear Dynamics, vol. 38, no. 1-4, pp. 369-381, 2004.

[51] Y. Q. Chen, H. Dou, B. M. Vinagre, and C. A. Monje, "A robust tuning method for fractional order PI controllers," in Proceedings of the 2nd IFAC Workshop on Fractional Differentiation and Its Applications, Porto, Portugal, 2006.

[52] C. A. Monje, B. M. Vinagre, V. Feliu, and Y. Chen, “Tuning and auto-tuning of fractional order controllers for industry applications," Control Engineering Practice, vol. 16, no. 7, pp. 798-812, 2008.

[53] Mathworks Inc., "Matlab optimization toolbox user's guide," 2007.

[54] V. Milanés, C. González, J. E. Naranjo, E. Onieva, and T. de Pedro, "Electro-hydraulic braking system for autonomous vehicles," International Journal of Automotive Technology, vol. 11, no. 1, pp. 89-95, 2010.

[55] V. Milanés, D. F. Llorca, B. M. Vinagre, C. González, and M. A. Sotelo, "Clavileno: evolution of an autonomous car," in Proceedings of the 13th IEEE International Intelligent Transportation Systems, Madeira, Portugal, 2010.

[56] Mathworks Inc., "Matlab identification toolbox user's guide," 2007.
[57] BECHTEL, "Compendium of executive summaries from the maglev system concept definition final reports," Tech. Rep., U.S. Department of Transportation, 1993, http://ntl.bts.gov/ DOCS/CES.html. 


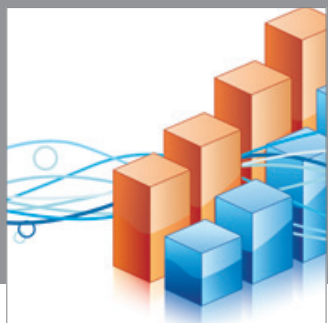

Advances in

Operations Research

mansans

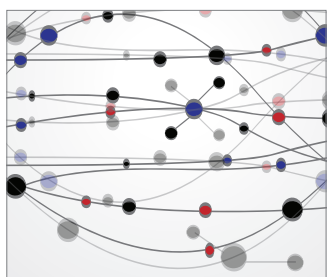

The Scientific World Journal
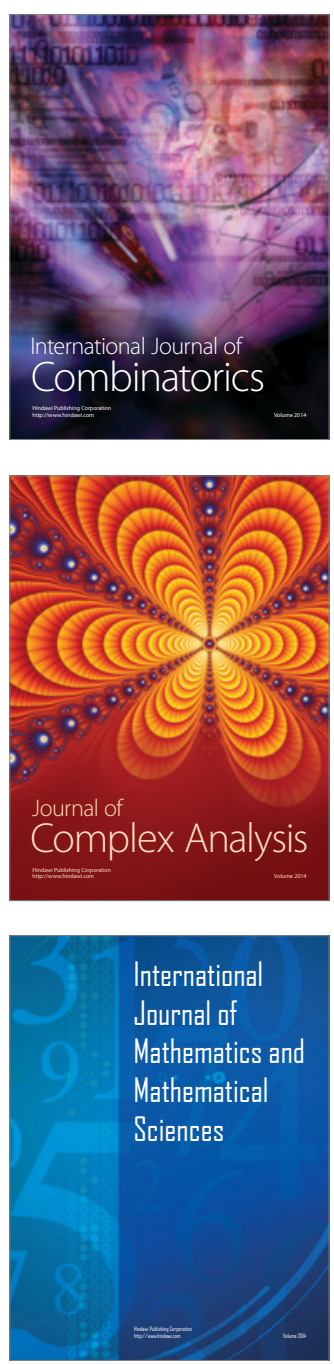
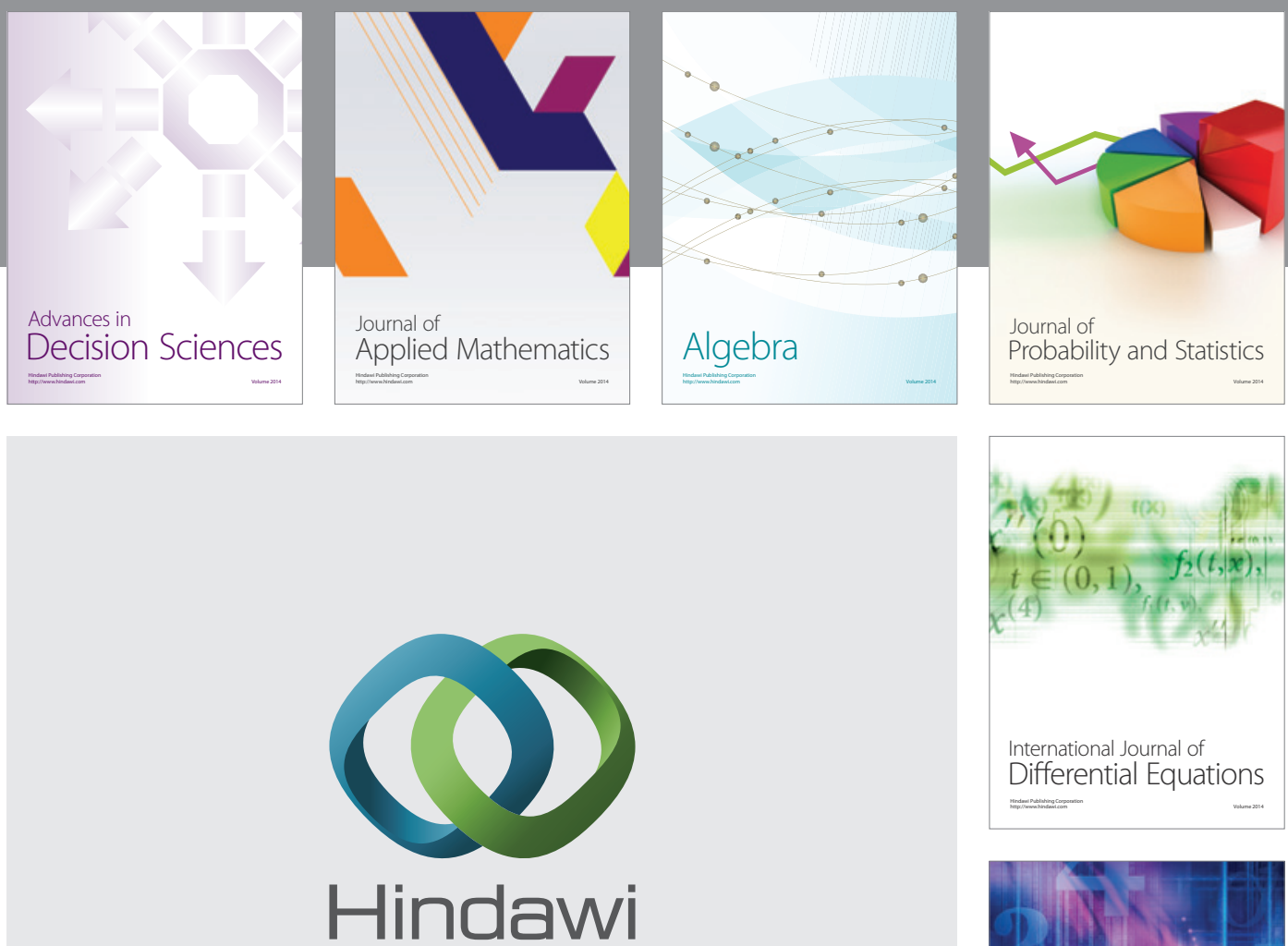

Submit your manuscripts at http://www.hindawi.com
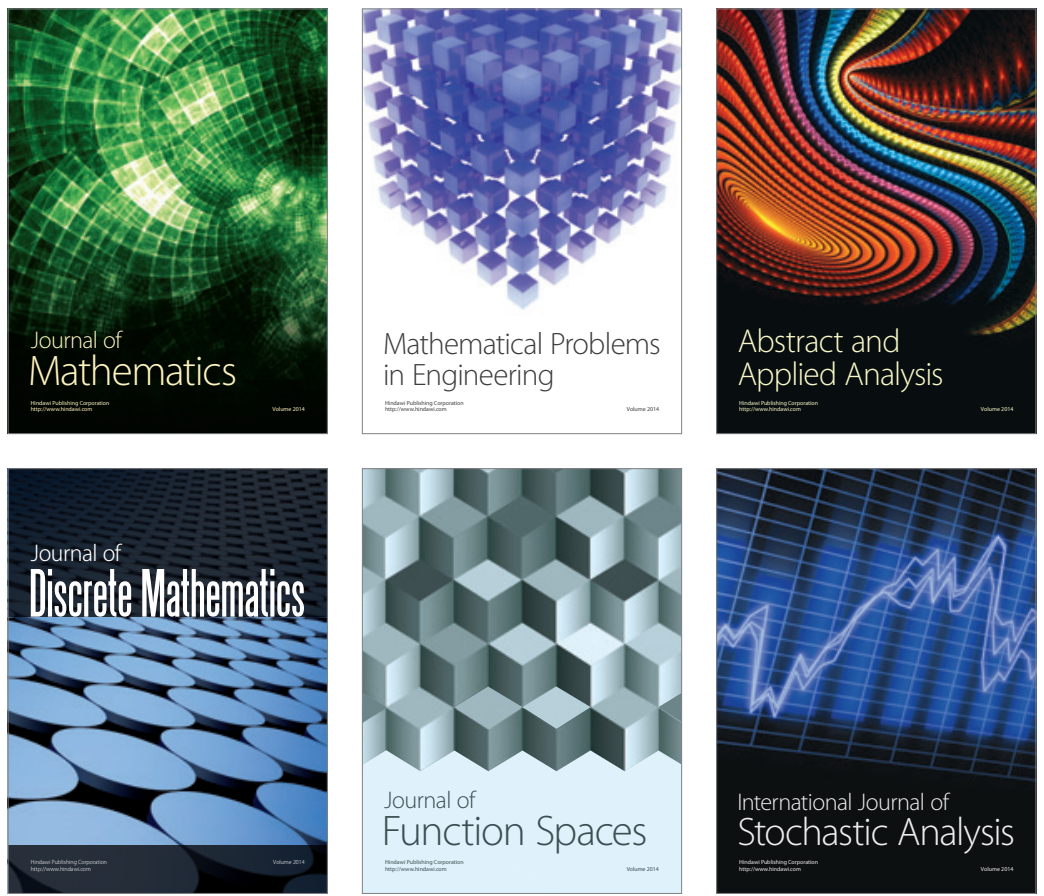

Journal of

Function Spaces

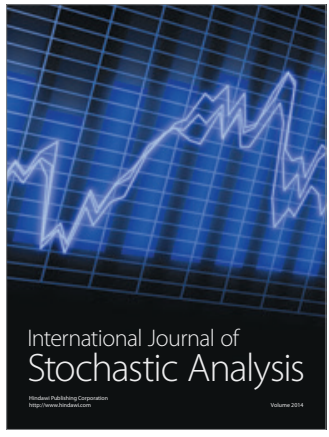

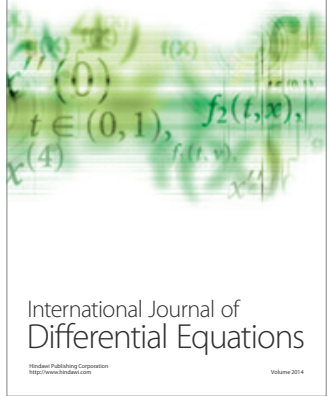
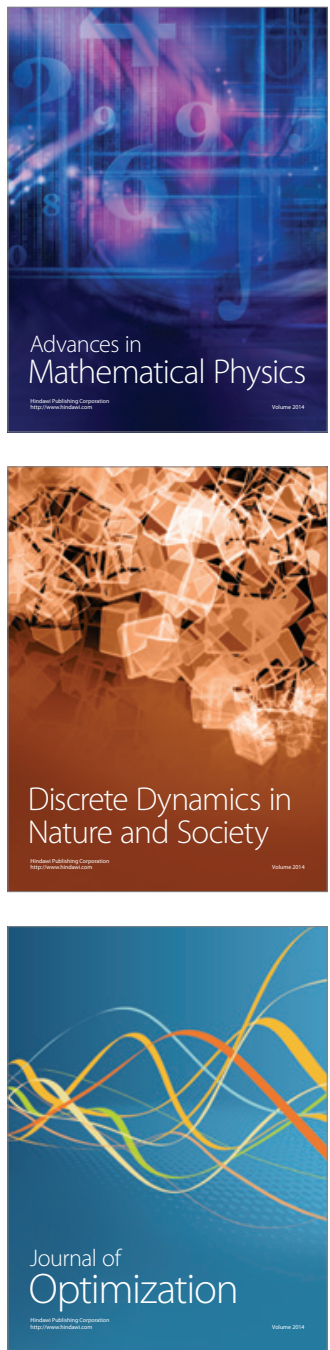